\title{
Publisher Correction: Subsets of ILC3-ILC1-like cells generate a diversity spectrum of innate lymphoid cells in human mucosal tissues
}

\author{
Marina Cella (D), Ramya Gamini, Cristiane Sécca (D), Patrick L. Collins, Shanrong Zhao, Vincent Peng, \\ Michelle L. Robinette $\mathbb{D}^{\mathbb{D}}$, Jorge Schettini, Konstantin Zaitsev (D), William Gordon, Jennifer K. Bando, Kentaro Yomogida, \\ Victor Cortez, Catrina Fronick, Robert Fulton, Lih-Ling Lin, Susan Gilfillan, Richard A. Flavell@, Liang Shan, \\ Maxim N. Artyomov, Michael Bowman, Eugene M. Oltz, Scott A. Jelinsky (1D and Marco Colonna (iD
}

Correction to: Nature Immunology https://doi.org/10.1038/s41590-019-0425-y, published online 17 June 2019.

In the version of this article initially published, there were several spacing, typographical and punctuation errors: Results, first subsection, fifth sentence, incorrect spacing (“...ILC3b,CD103+CD196+CD300LF- cellsasILC1bandCD103 ${ }^{+}$CD196-CD300LF ${ }^{-}$cells...”); Results, third subsection, first paragraph, third sentence, extraneous colon (“...indicative of: transforming growth factor...”); Results, fourth subsection, second sentence, incorrect second closing parentheses (“...neighbor embedding (t-SNE)) using...”); Fig. 6 title, incorrect final cell subset (“...ILC1b and ILC3a.”); Methods, eleventh subsection, first sentence, incorrect spacing (“...CD300LFand CD103...”); and Methods, twelfth subsection, second sentence, incorrect comma placement (“...protein-A-Mnase and chromatin, was...”). The correct text is as follows: Results, first subsection, fifth sentence, “...ILC3b, CD103+CD196 ${ }^{+}$CD300LF ${ }^{-}$cells as ILC1b and CD103+CD196 ${ }^{-} \mathrm{CD}^{+} \mathrm{CDOLF}^{-}$cells..."; Results, third subsection, first paragraph, third sentence, “...indicative of transforming growth factor...”; Results, fourth subsection, second sentence, “...neighbor embedding (t-SNE) using...”; Fig. 6 title, “...ILC1b and ILC1a."; Methods, eleventh subsection, first sentence, "...CD300LF and CD103..."; and Methods, twelfth subsection, second sentence, "...protein-A-Mnase, and chromatin was.... The errors have been corrected in the HTML and PDF versions of the article.

\section{Publisher Correction: Fat IL-33 sources}

Laurie A. Dempsey

Correction to: Nature Immunology https://doi.org/10.1038/s41590-019-0439-5, published online 18 June 2019.

In the version of this article initially published, an author name was misspelled ('Mallakõiv'). The correct spelling is 'Mahlakõiv'. The error has been corrected in the HTML and PDF versions of the article.

Published online: 6 August 2019

https://doi.org/10.1038/s41590-019-0475-1

\section{Author Correction: At the end of the beginning: immunotherapies as living drugs}

\author{
Ellen G. Feigal D, Natalie D. DeWitt, Cathy Cantilena, Carl Peck and David Stroncek
}

Correction to: Nature Immunology https://doi.org/10.1038/s41590-019-0452-8, published online 15 July 2019.

In the version of this article initially published, a word ('elimination') was incorrect in the third sentence of the final paragraph of the introduction, and the first word ('Immuno-oncology') in the title of the Fig. 2 legend was incorrect. The correct word in the introduction is 'attenuation'; the correct Fig. 2 title is "Approaches in various stages of development." The errors have been corrected in the HTML and PDF versions of the article. 